\title{
Decreased Vagal Influence on the Heart After 24-week Carnitine Supplementation
}

\author{
Stanislaw Zajaczkowski ${ }^{1}$, Piotr Badtke ${ }^{1}$, Angelika K Sawicka ${ }^{2}$, Milosz A Zajaczkowski ${ }^{3}$ \\ Robert A Olek ${ }^{2}$, Tomasz H Wierzba ${ }^{1}$ \\ ${ }^{1}$ Department of Physiology, Medical University of Gdansk, Gdansk, Poland \\ ${ }^{2}$ Department of Bioenergetics and Nutrition, Gdansk University of Physical Education and Sport, \\ Gdansk, Poland \\ ${ }^{3}$ Department of Clinical Anatomy, Medical University of Gdansk, Gdansk, Poland
}

\begin{abstract}
L-carnitine which shuttles fatty acid into the mitochondria is known diet supplement used by athletes to improve physical performance. It is also used in supplementary treatment in cardiac insufficiency. Since effect of L-carnitine on cardiovascular regulation is unclear we investigated whether its prolonged supplementation would affect autonomic nervous system (ANS) control upon the heart in healthy elderly volunteers.

Thirteen apparently healthy women aged 64 to 71 were supplemented in the double-blinded fashion with either $1.5 \mathrm{~g}$ of L-carnitine or placebo for 24 weeks. High resolution electrocardiogram was recorded before and after the supplementation. Time- and frequency-domain heart rate variability (HRV) analysis was performed to assess ANS control on the heart.

Following L-carnitine supplementation the overall HRV represented by TSP or SDNN insignificantly decreased with a significant reduction of parasympathetic-related HRV indexes: rMSSD, HF and SD1. Consistently, a tendency towards an increase of indexes of sympathovagal balance: SDNN/rMSSD and SD2/SD1 may reflect vagal withdrawal. Reduction of autonomic control on the heart after L-carnitine chronic supplementation may be regarded as its adverse effect related to reported increased risk of severe cardiac events.
\end{abstract}

\section{Introduction}

L-carnitine is a low-molecular amino acid derivative contributing in cellular energy balance. It plays a crucial role in fatty acid transport into mitochondria especially in myocardium [1] and skeletal muscle. Thus L-carnitine has been implemented in treatment of some cardiac pathologies [2] and for improvement of exercise performance. It has also been used for decades as a freely available dietary supplement [3]. Notwithstanding the large number of studies on functional effects of L-carnitine supplementation in skeletal muscle and heart, there is virtually no data about its regulatory relevance including the autonomic heart rhythm control.

In this study, we investigated the effect of 24-week L-carnitine supplementation on autonomic control of the heart in elderly people with no reported diseases. Heart rate variability (HRV) analysis is commonly used noninvasive method to assess autonomic nervous system (ANS) activity. It is well known that HRV is reduced with age [4-6], which correlates with increased risk of dangerous cardiovascular events. We hypothesized that L-carnitine supplementation might improve the ANS heart control.

\section{Materials and Methods}

Apparently healthy volunteers aged 64 to 71 responded to the advertisements in the local newspaper at the University of Third Age and at the Senior Activity Center. Subjects with cancer, cardiovascular, gastrointestinal, liver or renal diseases as well as with atrial fibrillation or frequent extrasystolic beats were excluded from the study. Exclusion criteria included prolonged treatment with thyroxine, beta-blockers, ACE inhibitors or calcium channel blockers. Thirteen females were finally enrolled and accomplished study. All individuals signed their consent to participate in the study. The study was conducted in accordance with the Declaration of Helsinki, and the experimental protocol was approved by the Independent Bioethics Commission for Research at Medical University of Gdansk (NKBBN/354-304/2015).

The participants were randomly divided into two groups: placebo $(\mathrm{N}=7)$ or L-carnitine $(\mathrm{N}=6)$ in the doubleblinded fashion. Subjects were receiving orally either 1500 $\mathrm{mg}$ of L-carnitine-L-tartrate or an isonitrogenous placebo per day for 24 weeks. Before the start and following the supplementation subjects arrived at the laboratory for tests [7] including electrocardiogram (ECG) recording. After a short initial relaxation (a few minutes) in supine position, high resolution $(4 \mathrm{kHz}) \mathrm{ECG}$ was recorded continuously for 10 to $20 \mathrm{~min}$ with the use of PowerLab 26T (AdInstruments, Sydney, Australia). 


\subsection{Heart Rate Variability measurements}

ECG recordings were visually checked to identify artifactual fragments or extrasystolic beats. Five-minute time-series of the best quality were selected from the original recordings. RR intervals (RRi) were identified using automatic R-peak detection of ECG (LabChart 8 Pro software, AdInstruments, Sydney, Australia). Additionally, sporadic false positive $\mathrm{R}$ wave detections, missing fragments or outstanding RRi were also manually corrected with use of Kubios Professional 3.2 software (Kubios Professional 3.2, Kuopio, Finland). The smooth priors method with $\lambda=500$ and interpolation rate $=4 \mathrm{~Hz}$ was used for smoothing data set prior to spectral analysis of HRV [8]. After correction of RRi-time-series to obtain normal-to-normal RR intervals (NN-time-series), HRV analysis was performed with use of Kubios Professional 3.2 software.

HR (heart rate), mean RRi, SDNN (the standard deviation of all normal NN intervals), rMSSD (square root of the mean of the squares of differences between adjacent $\mathrm{NN}$ intervals) and SDNN/rMSSD ratio were taken as a representative time-domain parameters. The frequencydomain parameters were assessed with fast Fourier transform (FFT) algorithm with estimation of spectral density using Welch's periodogram [9]. Power spectral densities were assessed from three 150 -second windows with $50 \%$ overlapping. Standard predefined spectral bands [10] were set at $0.04 \pm 0.15 \mathrm{~Hz}$ (low frequency, LF) and $0.15 \pm 0.4 \mathrm{~Hz}$ (high frequency, HF) and were expressed in absolute values $\left(\mathrm{ms}^{2}\right)$. Total spectral power (TSP) and LF/HF index were also calculated. Additionally SD1 and SD2 from Poincare plot as well as $\mathrm{SD} 2 / \mathrm{SD} 1$ index were assessed as representatives of non-linear HRV parameters.

Statistical analysis was performed using GraphPad Prism 7 software (La Jolla, CA, USA). All data sets were tested for normality with Shapiro-Wilk test. Statistical analysis was based on Mann-Whitney, Wilcoxon and paired or unpaired Student t-tests, depending whether data have normal or non-normal distribution. $\mathrm{P}$ values $<0.05$ were considered statistically significant. All data are shown as mean values \pm standard deviation of the mean $( \pm \mathrm{SD})$.
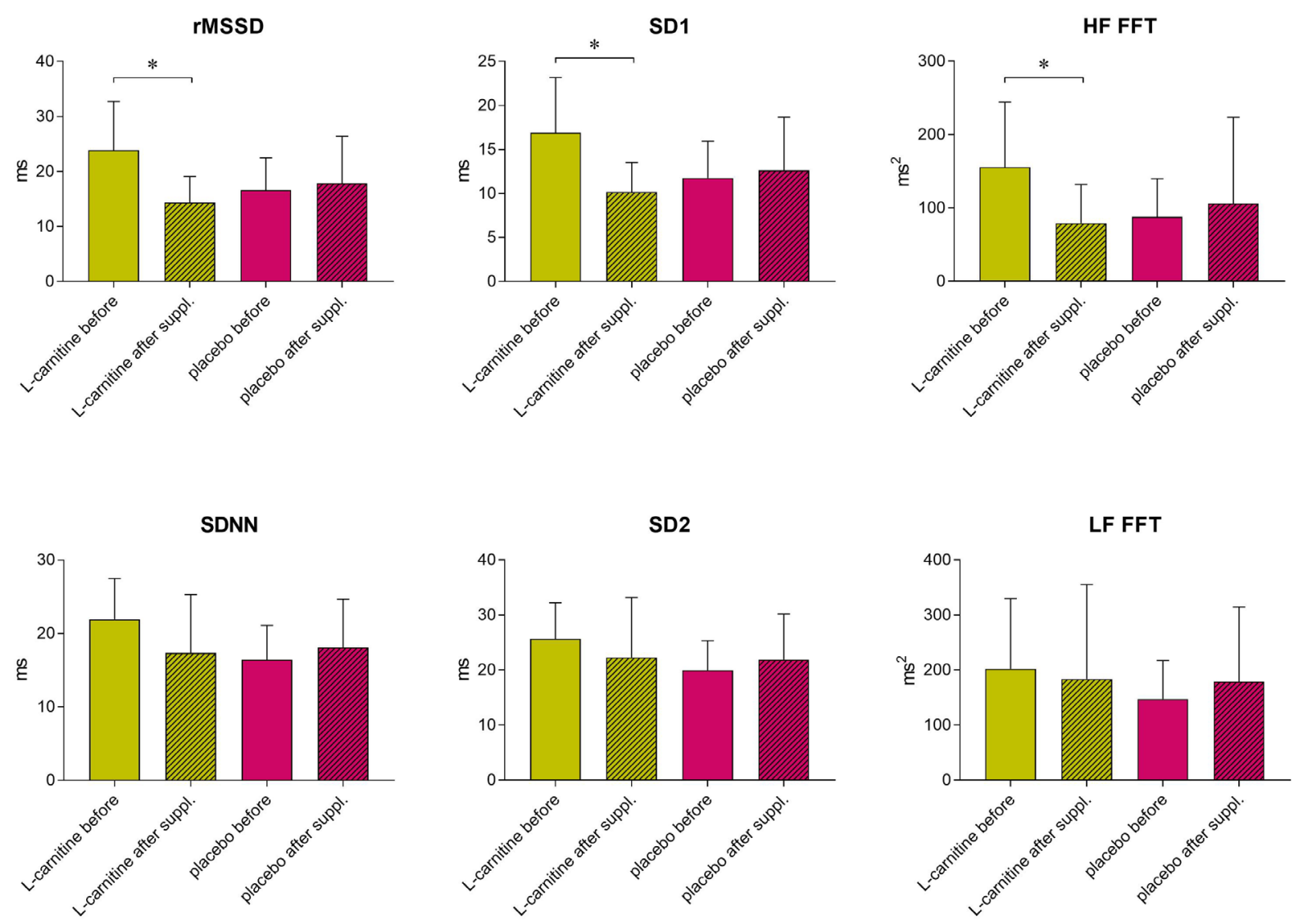

Figure 1. Selected time-domain and spectral HRV parameters before and after L-carnitine (green) or placebo (red) supplementation. $*$ - $p<0.05$; data are shown as mean \pm SD. Abbreviations are explained in text. 


\section{Results}

\subsection{Group characteristic}

As shown in Table 1 all participants did not differ in their mean age, body weight (BW) and body mass index (BMI). Either the body weight and the BMI were insignificantly higher in placebo group.

Table 1. Basic characteristic of the participants. Data are shown as mean $\pm \mathrm{SD}$. Abbreviations are explained in text.

\begin{tabular}{lccc}
\hline Parameter & Placebo & L-carnitine & $\mathrm{p}$ \\
\hline Age $($ years $)$ & $67 \pm 2$ & $67 \pm 3$ & 0.794 \\
BW $(\mathrm{kg})$ & $73.9 \pm 18.5$ & $7.2 \pm 6.6$ & 0.534 \\
BMI $\left(\mathrm{kg} / \mathrm{m}^{2}\right)$ & $29.8 \pm 6.8$ & $26.8 \pm 2.5$ & 0.534 \\
\hline
\end{tabular}

\subsection{Heart Rate Variability}

In L-carnitine group HR ranged between 60 and 85 beats per minute (bpm) before and from 65 to $86 \mathrm{bpm}$ after the L-carnitine supplementation (NS; $\mathrm{p}=0.063)$. In placebo group HR remained almost unchanged ranging from 58 to $81 \mathrm{bpm}$ before and from 56 to $79 \mathrm{bpm}$ after 24 weeks. Total HRV represented by TSP or SDNN showed insignificant decrease after L-carnitine supplementation. TSP changed from $405.3 \pm 211.1$ to $321 \pm 297$ (NS, p = $0.313)$ and SDNN from $21.91 \pm 5.59$ to $17.34 \pm 7.98 \mathrm{~ms}$ $(\mathrm{NS} ; \mathrm{p}=0.16)$. In placebo group we did not observed alike difference.

Before the L-carnitine supplementation we did not find any significant differences in HRV parameters related to vagal drive (rMSSD, SD1, HF) between placebo and Lcarnitine group (Figure 1). Following L-carnitine supplementation, but not in the placebo group, the vagal HRV indexes were suppressed: rMSSD (from $23.87 \pm 8.84$ $\mathrm{ms}$ to $14.35 \pm 4.72 \mathrm{~ms} ; \mathrm{p}=0.034$ ), SD1 (from $16.91 \pm 6.26$ $\mathrm{ms}$ to $10.16 \pm 3.34 \mathrm{~ms}, \mathrm{p}=0.034$ ) and HF (from $155.6 \pm$ $88.53 \mathrm{~ms}^{2}$ to $79.38 \pm 52.66 \mathrm{~ms}^{2}, \mathrm{p}=0.049$; Figure 1). In contrast to decreased short-term HRV parameters only irrelevant tendency towards a decrease of long-term HRV indexes were observed after L-carnitine supplementation: LF $202.3 \pm 127.6$ vs. $183.7 \pm 171.9 \mathrm{~ms}^{2}$, (NS, $\left.\mathrm{p}=0.71\right)$ and SD2 $25.68 \pm 6.52$ vs. $22.29 \pm 10.91 \mathrm{~ms}$, (NS, p $=0.31)$.
The tested indexes of sympathovagal balance: SDNN/rMSSD and SD2/SD1 showed an insignificant increasing tendency after L-carnitine supplementation (respectively $\mathrm{p}=0.078$ and $\mathrm{p}=0.067$ ). Interestingly $\mathrm{LF} / \mathrm{HF}$ index increased insignificantly in both groups (Table 2).

\section{Discussion}

Despite common use of L-carnitine as diet supplement routinely used for building of muscle mass and muscular strength and also as fat tissue burning [3] its regulatory effects are poorly understood. L-carnitine was reported to improve systolic function of the left ventricle in patients with heart failure [11], after acute myocardial infarction [12], reduce inflammation and fibrosis in coronary heart disease [13], improve cardiac electrophysiological parameters, ameliorate peripheral neuropathy in diabetes [14], improve body weight reduction during obesity treatment [15], and many more. L-carnitine, by its contribution in the transport of fatty acids through mitochondrial membrane, was thought not only to improve skeletal muscle and cardiac function in older people but also to support ANS regulatory control.

Surprisingly our results show that 24-week L-carnitine supplementation in elderly women evoked slight decline in total HRV and significant reduction in parasympathetic parameters such as: rMSSD, HF or SD1 (Figure 1). Observed changes accompanied by a minor HR increase and shifts of sympathovagal balance indexes towards sympathetic predominance (Table 2) might suggest withdrawal of vagal activity. A decrease in the autonomic function with aging, with shifting the sympathovagal balance towards sympathetic predominance is well-known phenomenon. Our data show that L-carnitine neither did not reduce the age-related sympathetic prevalence nor improve the overall ANS drive on the heart in healthy elderly people. Observed reduction of short-term HRV which is indicative for vagal suppression may be related to reported increased risk of sudden cardiac incidents [16]. The mechanism of such dysregulation is unclear. It is wellknown that nitric oxide (NO) affects ANS and can increase the vagal control of the heart [17]. On the other hand, Lcarnitine was reported to suppress NO production [18] despite its oxygen radical scavenging properties [19]. Perhaps antioxidant effects of L-carnitine, which like other antioxidants might cause increase in HRV [20], was attenuated by a suppression of NO.

Table 2. RR interval and all tested sympathovagal balance indexes of HRV before and after supplementation of L-carnitine or placebo. $\mathrm{P}$ close to significance is bolded. Abbreviations are explained in text.

\begin{tabular}{lcccccccc}
\hline \multicolumn{1}{c}{ Parameter } & $\begin{array}{c}\text { Placebo } \\
\text { before (1) }\end{array}$ & $\begin{array}{c}\text { Placebo after } \\
\text { suppl. (2) }\end{array}$ & $\begin{array}{c}\text { L-carnitine } \\
\text { before (3) }\end{array}$ & $\begin{array}{c}\text { L-carnitine } \\
\text { after } \\
\text { suppl.(4) }\end{array}$ & $\begin{array}{c}\mathrm{p} \\
\text { 1 vs 2 }\end{array}$ & $\begin{array}{c}\mathrm{p} \\
\text { 3 vs 4 }\end{array}$ & $\begin{array}{c}\text { p } \\
\text { 1 vs 3 }\end{array}$ & $\begin{array}{c}\mathrm{p} \\
\text { vs } 4\end{array}$ \\
\hline RRi (ms) & $905 \pm 102$ & $935 \pm 117$ & $901 \pm 108$ & $863 \pm 89.9$ & 0.313 & $\mathbf{0 . 0 5 7}$ & 0.954 & 0.250 \\
SDNN/rMSSD & $1.01 \pm 0.12$ & $1.06 \pm 0.26$ & $0.99 \pm 0.26$ & $1.21 \pm 0.21$ & 0.548 & $\mathbf{0 . 0 7 8}$ & 0.838 & 0.628 \\
SD2/SD1 & $1.76 \pm 0.28$ & $1.84 \pm 0.61$ & 1.69 \pm 0.59 & $2.14 \pm 0.34$ & 0.770 & $\mathbf{0 . 0 6 7}$ & 0.784 & 0.311 \\
LF/HF & $1.86 \pm 0.68$ & $2.53 \pm 2.01$ & Pagej3 1.63 & $2.46 \pm 1.06$ & 0.447 & 0.400 & 0.987 & 0.944 \\
\hline
\end{tabular}


Regrettably in vivo activity of NO was not assessed in this study. As older population exhibits low parasympathetic activity, any further reduction of vagal drive may likely result in significant suppressive effect on short-term HRV parameters.

\section{Summary}

L-carnitine supplementation in healthy elder people did not improve ANS control of the heart. Moreover, it caused a decrease in vagal nerve activity which reportedly is an unfavorable effect.

\section{Acknowledgments}

The study was supported by National Science Center 2014/15/B/NZ7/00893 and by Ministry of Science and Higher Education for young scientists 01-0336/08/261.

\section{References}

[1] L. H. Opie, "Role of carnitine in fatty acid metabolism of normal and ischemic myocardium," Am Heart J, no. 97; 3, pp. 375-388, 1979.

[2] M. A. Arsenian, "Carnitine and its derivatives in cardiovascular disease," Prog Cardiovasc Dis, vol. 40, no. 3, pp. 265-286, 1997.

[3] F. B. Stephens et al., "Skeletal muscle carnitine loading increases energy expenditure, modulates fuel metabolism gene networks and prevents body fat accumulation in humans," J Physiol, vol. 591, no. 18, pp. 4655-4666, 2013.

[4] J. B. Schwartz, W. J. Gibb, and T. Tran, "Aging effects on heart rate variation," J Gerontol, vol. 46, no. 3, M99106, 1991.

[5] N. Craft and J. B. Schwartz, "Effects of age on intrinsic heart rate, heart rate variability, and AV conduction in healthy humans," Am J Physiol, vol. 268, no. 4 Pt 2, H1441-52, 1995.

[6] S. Zajączkowski, M. A. Zajączkowski, A. Kosiński, M. Grzybiak, and T. H. Wierzba, "Ocena zmienności rytmu serca (HRV) w zaawansowanym wieku. Heart rate variability (HRV) analysis in advanced age," Geriatria, no. 8, pp. 232-239, 2014.

[7] A. K. Sawicka et al., "L-carnitine supplementation in older women. A pilot study on aging skeletal muscle mass and function," Nutrients, vol. 10, no. 2, 2018.

[8] J. S. Gąsior et al., "Normative values for heart rate variability parameters in school-aged children: simple approach considering differences in average heart rate," Front Physiol, vol. 9, p. 1495, 2018.

[9] S. Zajaczkowski, M. Smolinska, P. Badtke, and T. H. Wierzba, "Time-domain and spectral analysis of heart rate variability in rats challenged with hypoxia," Comput Cardiol, no. 41, pp. 785-788, 2014.
[10] "Heart rate variability: standards of measurement, physiological interpretation and clinical use. Task force of the European society of cardiology and the north American society of pacing and electrophysiology," Circulation, vol. 93, no. 5, pp. 1043-1065, 1996.

[11] Y. Omori et al., "L-Carnitine prevents the development of ventricular fibrosis and heart failure with preserved ejection fraction in hypertensive heart disease," $J$ Hypertens, vol. 30, no. 9, pp. 1834-1844, 2012.

[12] J. J. DiNicolantonio, C. J. Lavie, H. Fares, A. R. Menezes, and J. H. O'Keefe, "L-carnitine in the secondary prevention of cardiovascular disease: systematic review and meta-analysis," Mayo Clin Proc, vol. 88, no. 6, pp. 544-551, 2013.

[13] B.-J. Lee, J.-S. Lin, Y.-C. Lin, and P.-T. Lin, "Antiinflammatory effects of L-carnitine supplementation $(1000 \mathrm{mg} / \mathrm{d})$ in coronary artery disease patients," Nutrition, vol. 31, no. 3, pp. 475-479, 2015.

[14] H. Ulvi, R. Aygül, and R. Demir, "Effect of L-carnitine on diabetic neuropathy and ventricular dispersion in patients with diabetes mellitus," Turk J Med Sci, no. 40 (2), pp. 169-175, 2010.

[15] M. Pooyandjoo, M. Nouhi, S. Shab-Bidar, K. Djafarian, and A. Olyaeemanesh, "The effect of (L-)carnitine on weight loss in adults: a systematic review and metaanalysis of randomized controlled trials," Obes Rev, vol. 17, no. 10, pp. 970-976, 2016.

[16] L. Carnevali et al., "Low vagally-mediated heart rate variability and increased susceptibility to ventricular arrhythmias in rats bred for high anxiety," Physiol Behav, vol. 128, pp. 16-25, 2014.

[17] S. Chowdhary and J. N. Townend, "Role of nitric oxide in the regulation of cardiovascular autonomic control," Clin Sci, vol. 97, no. 1, pp. 5-17, 1999.

[18] A. Koc, T. Ozkan, A. Z. Karabay, A. Sunguroglu, and F. Aktan, "Effect of L-carnitine on the synthesis of nitric oxide in RAW $264 \cdot 7$ murine macrophage cell line," Cell Biochem Funct, vol. 29, no. 8, pp. 679-685, 2011.

[19] P. J. Arockia Rani and C. Panneerselvam, "Carnitine as a free radical scavenger in aging," Exp Gerontol, vol. 36, no. 10, pp. 1713-1726, 2001.

[20] S. Zajączkowski et al., "Promising effects of xanthine oxidase inhibition by allopurinol on autonomic heart regulation estimated by heart rate variability (HRV) analysis in rats exposed to hypoxia and hyperoxia," PLoS $O N E$, vol. 13, no. 2, 2018.

Corresponding address:

Stanislaw Zajaczkowski, PhD

Medical University of Gdansk

Department of Physiology

1 Debinki Street

80-211 Gdansk, Poland

s.zajaczkowski@gumed.edu.pl 